\title{
IL CHIOSTRO DI SAN BARTOLOMEO A LIPARI: SPERIMENTAZIONI PROGETTUALI E DECORATIVE NELLA PRIMA COMUNITÀ BENEDETTINA DELLA SICILIA NORMANNA
}

\author{
MARGHERITA TABANELLI
}

UDC: $726.71(450.82) " 11 "$

Original scientific paper

Manuscript received: 24. 10. 2016.

Revised manuscript accepted: 07. 02. 2017.

DOI: 10.1484/J.HAM.5.113723
M. Tabanelli

Via Trieste 939

48123 Marina di Ravenna (RA)

Italia

The most ancient Norman Benedictine abbey in Sicilian area is S. Bartolomeo in Lipari. Count Roger I and his brother Robert Guiscard established it a couple of years before the death of the latter in 1085. In 1767, an earthquake damaged the church, which was rebuilt as a three naves basilica. The current right aisle has replaced the northern side of the cloister. The cloister is probably the oldest one in Southern Italy. The quality of the capitals' sculptures, as well as the lack of narrative scenes, attests that the cloister was built some decades after the abbey's foundation, during the first half of the $12^{\text {th }}$ century. The style of the sculptures has no comparisons in the Norman world, but it is possible to find similarities with the First-Romanesque production of Northern and Central Italy. The artists likely were part of the community of Lombard colonists invited in Sicily by count Roger and his wife Adelasia, as well as the first abbot, Ambrosius, and many monks. Both architecture and decoration of the monastic complex seem to have adapted themselves to the habits and the expectations of the first community of Lombard Benedictines, instead to them of their Norman patrons.

Keywords: Lipari, S. Bartolomeo, Cathedral, Normans, Cloister, $12^{\text {th }}$ century Architecture, Sculpture, Benedictines, Sicily.

\section{UN MONASTERO BENEDETTINO A PRESIDIO DELLE EOLIE}

A oltre un ventennio di distanza dall'apertura delle ostilità contro gli Arabi, la conquista della Sicilia era ancora ben lontana da una conclusione ${ }^{1}$ e il Val di Noto restava sotto il controllo dell'emiro di Siracusa Benarvet, coadiuvato dai rinforzi giunti dall'Ifriqiya per ordine del sovrano zirita Temîm². Il quadro si aggravò per Ruggero I Altavilla nei primi anni ottanta dell'XI secolo, quando la defezione del figlio Giordano minò la stabilità di un'area, il Val Demone, occupata dai Normanni già da due decenni ${ }^{3}$. Le sponde dello Stretto furono al contempo bersaglio di incursioni zirite, che colpirono con particolare intensità il centro fortificato di Nicotera, posto a guardia del tratto di costa ai piedi della sede comitale di Mileto ${ }^{4}$. Non desta meraviglia, quindi, la scelta dei fratelli Altavilla di istituire un monastero benedettino a Lipari, nell'intento di consolidare la presenza normanna nello spopolato ma strategico arcipelago eoliano.
Le isole, dopo aver conosciuto una relativa floridezza fino alla tarda età romana ${ }^{5}$, erano state turbate tra VIII e IX secolo dalla riattivazione del vulcano liparese del Monte Pelato e impoverite dalle depredazioni e deportazioni aglabidi dell' 835 e $838^{6}$. Dalla desolata Lipari il principe longobardo Sicardo sottrasse le ossa di san Bartolomeo poco dopo il passaggio dei Saraceni, portandole prima a Salerno e quindi a Benevento, dove furono accolte in una nuova chiesa eretta accanto alla cattedrale? .

Che all'arrivo dei normanni l'arcipelago fosse scarsamente popolato lo attestano gli sforzi di attrazione di nuovi coloni attraverso la concessione di terreni a vantaggiose condizioni, di cui resta traccia per l'isola maggiore nel constitutum dell'abate Ambrogio ${ }^{8}$. La comunità benedettina si era insediata a Lipari entro il 1085, recuperando l'antica dedica a San Bartolomeo, e aveva ottenuto in possesso tutte le Eolie ${ }^{9}$. Nei piani del Gran Conte la rivitalizzazione non avrebbe dovuto limitarsi all'insediamento monastico già

${ }^{1}$ Le operazioni militari furono avviate a poca distanza dalla conquista della Calabria, nel 1061: M. AMARI, Storia dei musulmani di Sicilia, Firenze, 2, 18541868, p. 550-552; F. CHALANDON, Histoire de la domination normande en Italie et en Sicile, 1, Paris, 1907, p. 192-194; J. BECKER Graf Roger I. von Sizilien: Wegbereiter des normannischen Königreichs, Tübingen, 2008, p. 48-50.

${ }^{2}$ M. AMARI, op. cit. (n. 1), 3, p. 109-111; F. CHALANDON, op. cit. (n. 1), 1, p. 327-334; J. BECKER, op. cit. (n. 1), p. 62-63.

${ }^{3}$ F. CHALANDON, op. cit. (n. 1), 1, p. 335-340; J. BECKER, op. cit. (n. 1), p. 64-67.

${ }^{4}$ Sulla concessione di Mileto a Ruggero nei primi tempi della conquista: F. CHALANDON, op. cit. (n. 1), 1, p. 154; J. BECKER, op. cit. (n. 1), p. 44-45; sul ruolo della città al tempo della Contea: G. Occhiato (ed.), Ruggero I e la "provincia melitana", Soveria Mannelli, 2001. Sulle distruzioni di Nicotera nel 1074 e nel 1085: M. AMARI, op. cit. (n. 1), 3, p. 109-111.

${ }_{5}^{5}$ Sulle testimonianze archeologiche della Lipari greca e romana: L. BERNABÒ BREA, Le Isole Eolie dal Tardo Antico ai Normanni, Ravenna, 1988, p. 53-119. ${ }^{6}$ M. AMARI, op. cit. (n. 1), 1, p. 315-316; L. BERNABÒ BREA, op. cit. (n. 6), p. 7-18, 43-44, 125-129. Solleva dubbi sull'entità dei fenomeni vulcanici, anticipandoli inoltre alla seconda metà del VI secolo E. KISLINGER, La storia di Lipari bizantina riconsiderata, in V. GIUSTOLISI (ed.), Alla ricerca di Lipari bizantina, Palermo, 2001, p. 13-17.

7 Per una panoramica delle fonti sulla traslazione delle reliquie L. BERNABÒ BREA, op. cit. (n. 5), p. 18-28.

${ }^{8}$ L. CATALIOTO, Il vescovato di Lipari-Patti in età normanna (1088-1194). Politica, economia, società in una sede monastico-episcopale della Sicilia, Messina, 2007, p. 179-180, nr. 6. Il constitutum del 1095 rappresenta in realtà una stretta sulle nuove concessioni, ma consente di ricavare informazioni sulle norme precedentemente applicate.

9 Una donazione di Roberto il Guiscardo (m. 1085) fu confermata da Ruggero II nel 1134 insieme al possesso di Lippariam, Vulcanum, Salinam, Panaream, Strongelon, Arcudi et Filicudi insulas cum omnibus earum pertinentiis: L. CATALIOTO, op. cit. (n. 8), p. 206-208, nr. 39. La bolla di Urbano II del 1091 menziona tuttavia la sola Lipari. Garufi ritiene di poter stabilire la data di fondazione al 1083 (C. A. GARUFI, Per la storia dei secoli XI e XII, I. Le Isole Eolie. A proposito del Constitutum dell'Abate Ambrogio del 1095. Studi e ricerche, in Archivio storico per la Sicilia orientale, 9, 1912, 1, p. 167), ma l'ipotesi è stata 
custode del corpo dell'apostolo, bensì comprendere la sede episcopale associando le cariche di vescovo e abate come nel contemporaneo caso di Catania ${ }^{10}$. Lo attesta la bolla di papa Urbano II rilasciata il 3 giugno 1091, che conferma la nomina abbaziale di Ambrogio ma rifiuta di ricostituire la diocesi eoliana per via della «loci exiguitas» e della «accolarum raritas», pur riconoscendone l'esistenza in antico" ${ }^{11}$.

L'alto valore attribuito al monastero liparitano emerge anche dalle numerose donazioni di cui fu beneficiario nei primi decenni dopo la fondazione: non solo Roberto il Guiscardo e Ruggero I elargirono terreni e villani in diversi punti della Sicilia e della Calabria ${ }^{12}$, ma molti esponenti della nobiltà normanna concessero de rebus suis generosi lasciti. La maggioranza di essi si concentra tra il 1093 e il $1111^{13}$, mentre del decennio seguente calarono decisamente ritmo e consistenza delle donazioni ${ }^{14}$.

L'esaurirsi della fase di incremento patrimoniale coincise tuttavia con il raggiungimento dell'ambita promozione a sede vescovile, concessa nel 1131 dall'antipapa Anacleto II nella cornice di un più vasto riordino del sistema diocesano dell'isola ${ }^{15}$. La necessità di assicurarsi il sostegno di Ruggero II, ormai divenuto re della vasta compagine territoriale normanna, fornì motivazioni sufficienti per superare gli ostacoli evidenziati quarant'anni prima da Urbano II. D’altronde il territorio sottoposto a San Bartolomeo si era in quel lasso di tempo sensibilmente dilatato, grazie all'unione con il monastero di San Salvatore a Patti nel $1094^{16}$. Allo stesso tempo la politica di ripopolamento delle Eolie aveva dato rapidamente frutti, tanto che nel 1095 Ambrogio aveva rivisto le condizioni di concessione dei terreni per i nuovi coloni, riducendone decisamente la convenienza rispetto a quanto riconosciuto agli «antiqui habitatores» ${ }^{17}$.

L'origine dei coloni, che nel constitutum di Ambrogio per la comunità di Patti vengono definiti «homines quicumque sint Latine lingue $»^{8}$, non è stata finora oggetto di uno studio specifico, ma se ne è spesso ipotizzata la provenienza dalle terre soggette agli Aleramici, in analogia con il noto fenomeno degli oppida lombardorum ${ }^{19}$. Da un'analisi degli antroponimi presenti nelle pergamene ${ }^{20}$ sembra tuttavia di poter restituire un quadro più vario della nuova compagine etnica eoliana e pattese. La comunità monastica di San Bartolomeo era composta da religiosi sia di origine franco-normanna che longobarda e forse "lombarda", attribuendo a questo termine un'accezione ampia in riferimento all'area nordoccidentale della Penisola. Oltre all'abate Ambrogio, anche altri monaci firmatari di documenti portano nomi che non trovano riscontro nell'onomastica normanna ${ }^{21}$ : Adelmo ${ }^{22}$, Sigulfo, ${ }^{23}$ Anselmo, Lamberto,

rigettata da F. CHALANDON, op. cit. (n. 1), 1, p. 335 e L.T. WHITE, Latin monasticism in Norman Sicily, Cambridge, 1938 (ed. it. Il monachesimo latino nella Sicilia normanna, Catania, 1984), p. 123-124, che ridimensionano anche il ruolo del Guiscardo. Il primo documento noto è un diploma di conferma del possesso di alcuni terreni presso Mileto rilasciato da Ruggero I nel luglio 1088: L. CATALIOTO, op. cit. (n. 8), p. 173, nr. 1.

${ }^{10} \mathrm{Nel} 1091$ Ruggero nominò il bretone Ansgerio vescovo di Catania e abate del monastero benedettino annesso alla cattedrale: J. BECKER, op. cit. (n. 1), p. 176-181.

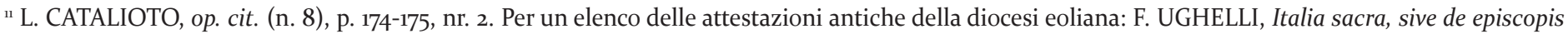
Italiae et insularum adiacentium, 1, Venetiis 1717, p. 771-775; R. PIRRI, Sicilia sacra disquisitionibus et notitiis illustrata, II ed., Panormi, 1733, 2, p. 948-952. V. anche H. ENZENSBERGER, Lipari come sede vescovile, in G.M. Bacci, M.A. Mastelloni (ed.), Alle radici della cultura mediterranea ed europea. I Normanni nello Stretto e nelle isole Eolie, Palermo, 2004, p. 20-22.

${ }^{12}$ L. CATALIOTO, op. cit. (n. 8), p. 181-182, nr. 9; J. BECKER, Documenti latini e greci del conte Ruggero I di Calabria e Sicilia, Roma, 2013, p. 76-77, nr. 12; p. 162-164, nr. 39; p. 165-168, nr. 40, p. 269-270, nr. 71.

${ }_{13}^{13}$ Il diploma di Ruggero del 1093 conferma anche le donazioni dei baroni Ruggero de Barneville, Guglielmo Mallabret; Goffredo Borrell, Riccardo Bonell, Amelino Gastinel, Roberto de Brocato, Goffredo de Sageio, Ruggero di Marchisio, Radolfo Bonell, Ruggero di Sourval, Odaldo di Calatasibet [J. BECKER, op. cit. (n. 12), p. 165-168, nr. 40]. Altri benefici furono concessi dal vescovo Roberto di Messina e Troina nel 1094 [L. CATALIOTO, op. cit. (n. 8), p. 178-179, nr. 5]; dalla figlia di Ruggero I Matilde e dal marito Roberto de Auceto del 1095 (ibidem, p. 180-181, nr. 7); Roberto Mandaguerra, Adamo d'Angiò, Guglielmo Malet, Stefano Malcovenant e Salomone figlio di Guido entro il 1098 [J. BECKER, op. cit. (n. 12), p. 245-246, nr. 64]; Ugo di Craon nel 1105 [L. CATALIOTO, op. cit. (n. 8), p. 184, nr. 12]; Achino di Vizzini nel 1106 (Ibidem, p. 185, nr. 13); Rainaldo, Fredesenda, Roberto e Drogone Avenel nel 1111 (Ibidem, p. 186-187, nr. 15). ${ }_{14}^{14}$ Due donazioni di Adelasia si collocano nel 1108 (Ibidem, p. 185-186, nr. 14) e tra 1111 e 1115 (Ibidem, p. 189, nr. 18); quella di Ruggero II nel 1118 (Ibidem, p. 190-191, nr. 20). Si segnala anche la concessione di un terreno presso Butera da parte di Enrico del Vasto nel 1130 (C.A. GARUFI, Gli Aleramici e $i$ Normanni in Sicilia e nelle Puglie, in Centenario della nascita di Michele Amari, 1, Palermo, 1910, p. 71, nr. 3).

${ }^{15}$ L.T. WHITE, op. cit. (n. 9), p. 89-91; N. KAMP, Der unteritalienische Episkopat im Spannungsfeld zwischen monarchischer Kontrolle und römischer "libertas" von der Reichsgründung Rogers II. bis zum Konkordat von Benevent, in Società, potere e popolo nell'età di Ruggero II, Atti delle terze Giornate normannosveve (Bari, 23-25 maggio 1977), Bari, 1979, p. 109-121; G. LOUD, The Latin Church in Norman Italy, Cambridge, 2007, p. 159-160 e 225-226; L'istituzione dell'episcopato si ricava dalla bolla di Anacleto II del 14 settembre 1131 che assegnava il vescovo di Lipari-Patti Giovanni quale suffraganeo all'arcivescovo di Messina Ugo [P.F. KEHR, Italia Pontificia, 10, Calabria-Insulae, Turici, 1975 (Regesta Pontificum Romanorum), p. 357, nr. 4].

${ }^{16}$ J. BECKER, op. cit. (n. 12), p. 162-164, nr. 39.

${ }^{17}$ C. A. GARUFI, op. cit. (n. 9); L. CATALIOTO, op. cit. (n. 8), p. 179-180, nr. 6. Il documento del 1095 venne riconfermato dal successore di Ambrogio, Giovanni, nel 1133: Ibidem, p. 203-204, nr. 36. Un ulteriore constitutum fu emanato da Ambrogio per i coloni di Patti, il cui testo è noto grazie alla trascrizione in una sentenza di Ruggero II del 1133: Ibidem, p. 200-202, nr. 33.

${ }^{18}$ L. CATALIOTO, op. cit. (n. 8), p. 201, nr. 33.

${ }_{19}$ C.A. GARUFI, op. cit. (n. 14); E. PONTIERI, La madre di Re Ruggero: Adelasia del Vasto contessa di Sicilia regina di Gerusalemme (?-1118), in ID., Tra i normanni dell'Italia meridionale, Napoli, 1964, p. 415-436; H. BRESC, Gli Aleramici in Sicilia: alcune nuove prospettive, in R. Bordone (ed.), Bianca Lancia d'Agliano. Fra il Piemonte e il Regno di Sicilia, Atti del convegno (Asti-Agliano, 28-29 aprile 1990), Alessandria, 1992, p. 147-173; L. CATALIOTO, op. cit. (n. 8), p. 147-169.

${ }^{20}$ Ibidem, Appendice A e C.

${ }^{21}$ L. R. MÉNAGER, Inventaire des familles normandes et franques émigrées en Italie méridionale et en Sicile (XIe-XII siècles), in Roberto il Guiscardo e il suo tempo, Relazioni e comunicazioni nelle Prime Giornate normanno-sveve (Bari, maggio 1973), Roma, 1975, p. 259-390; ID., Addition à l'inventaire des familles normandes et franques émigrées en Italie méridionale et en Sicile, in ID., Hommes et institutions de l'Italie normande, London, 1981, (Variorum Collected Studies Series, 136), p. 1-12; G. LOUD, op. cit. (n. 15), p. 120-125.

${ }^{22}$ L. CATALIOTO, op. cit. (n. 8), p. 176, nr. 3 (1093).

${ }_{23}$ Ibidem, p. 18o, nr. 6 (9 maggio 1095). 
Gandolfo ${ }^{24}$, nonché un «Philippum monacum arabum» ${ }^{25}$. Nel caso dei testimoni esterni alla comunità benedettina si incappa saltuariamente in indicazioni di provenienza: così testimone della donazione di Ruggero I del 1093 è un certo «Ildebrandus lombardus ${ }^{26}$, mentre nel 1133 a rappresentare l'episcopato eoliano nella causa contro gli abitanti di Patti è un «Donadeus Genuensis» ${ }^{27}$.

Poco convincente è invece l'interpretazione del nome del secondo abate di Lipari, Giovanni de Pergana, quale prova di una sua presunta origine bergamasca ${ }^{28}$. Questi è documentato quale successore di Ambrogio dal 1121 al 1148 e fu quindi il primo a beneficiare del titolo vescovile ${ }^{29}$. L'affermazione di Innocenzo II si riverberò sulle sorti dell'episcopato eoliano, la cui creazione fu dichiarata nulla dal Concilio Lateranense del $1139^{30}$. In conseguenza di ciò, seppur con un ritardo che denuncia una certa resistenza alle decisioni romane, Giovanni ritornò a sottoscrivere i documenti successivi al febbraio 1142 con il semplice titolo di abate ${ }^{31}$. Lo stesso vale per i suoi immediati successori Alessandro (1152), Osberno (1153-1155) e Gilberto (1156-1170), tutti designati dalle pergamene tuttalpiù come eletti ${ }^{32}$. Risale soltanto al 1171 la prima attestazione nota del ripristinato titolo episcopale, in riferimento a Pietro (1171-1174) ${ }^{33}$.

Con il passare del tempo fu invece l'elemento monastico a venir meno, cosicché la comunità benedettina, ancora citata nelle condizioni per la resa dell'isola a Roberto d'Angiò nel 1339, risultava già scomparsa al momento della costituzione di Lipari in diocesi autonoma da Patti nel 139934. Tale circostanza rappresentò, insieme all'incendio scaturito dall'assedio del pirata Kaireddin Barbarossa nel luglio $1544^{35}$, una concausa della perdita di funzionalità del chiostro e quindi della progressiva obliterazione delle sue strutture. Forse intorno alla metà del Seicento il braccio orientale fu convertito in cappella funeraria, mentre in un momento imprecisato segmentando il restante ambulacro si ricavarono numerosi piccoli ambienti posti in collegamento con il retrostante palazzo vescovile. L'aula aderente al transetto destro, verosimilmente l'antica sala capitolare, già prima dell'inizio del Seicento era stata trasformata in cappella sfondando il setto che la separava dalla chiesa e fu dotata di una nuova copertura e ridecorata dal vescovo Candido entro il $1645^{36}$. Maggiori sconvolgimenti furono dettati dal sisma del 1768 , in occasione del quale «tota corruit Cathedralis Ecclesia»37. Il vescovo Prestandrea affermava nel 1775 di aver completato la ricostruzione del corpo longitudinale, ampliato da due navate laterali, il che comportò la soppressione di quel che rimaneva del braccio settentrionale del chiostro e delle prime campate delle corsie attigue ${ }^{38}$.

Il presente contributo è dedicato all'organismo claustrale e alla sua decorazione, tralasciando per ragioni di spazio l'analisi dell'architettura della chiesa, sulla quale peraltro si ha avuto modo di ragionare in altra sede ${ }^{39}$. Sarà sufficiente ricordare che fino al 1768 la cattedrale si presentava come una chiesa mononave con transetto emergente e tre absidi orientate, presumibilmente coperta con un sistema di volte a botte e una cupola o tiburio d'incrocio. Le strutture monastiche rimasero camuffate da rifacimenti e tramezzi moderni fino agli Novanta, quando il completamento dei lavori di liberazione intrapresi un ventennio prima da Luigi Bernabò Brea riportò alla vista il più antico chiostro siciliano finora noto ${ }^{40}$.

\section{GLI AMBIENTI MONASTICI}

Resti del monastero sono ancora individuabili intorno ai tre lati conservati del chiostro, facilmente riconoscibili per le ordinate cortine murarie in grossi blocchi isodomi di pietra lavica, recuperati probabilmente dalle mura della città greco-romana ${ }^{41}$ (fig. 1). A est, la cappella del Santissimo Sacramento ha riutilizzato i perimetrali di un fabbricato rettangolare originariamente a due piani, contiguo a un corpo scala. Alla scala si accedeva sia dal chiostro, tramite un

\footnotetext{
${ }^{24}$ Ibidem, p. 93, nr. 23 (30 marzo 1125).

${ }^{25}$ Ibidem, p. 19o, nr. 19 (8 luglio 1117).

${ }^{26}$ Ibidem, p. 176, nr. 3 (1093).

${ }^{27}$ Ibidem, p. 202, nr. 33 (10 gennaio 1133).

${ }^{28}$ G. IACOLINO, La società eoliana nell'età della rinascenza, in G.M. Bacci, M.A. Mastelloni (ed.), op. cit. (n. 11), p. 70.

${ }^{29}$ L. CATALIOTO, op. cit. (n. 8), p. 79-88.

${ }^{30}$ N. KAMP, op. cit. (n. 15), p. 116-119.

${ }^{31}$ L. CATALIOTO, op. cit. (n. 8), p. 88-89 e 211-222.

${ }^{32}$ Ibidem, p. 88-89, 112-119 e 222-224.

33 L.T. WHITE, op. cit. (n. 9), p. 417-418, nr. 27; L. CATALIOTO, op. cit. (n. 8), p. 89-9o e 224-226.

${ }^{34}$ Sulla resa dei Liparesi: G.B. SIRAGUSA, Le imprese angioine in Sicilia, in Archivio Storico Siciliano, 15, 189o, p. 312-314. Il testo del documento di Bonifacio IX del 1399 è edito in R. PIRRI, op. cit. (n. 11), 2, p. 956-957.

35 G. IACOLINO, I Turchi alla Marina di Lipari: 1544. Con edizione critica e commento de La destruttione de Lipari per Barbarussa composta per Giovan Andria di Simon detto il Poeta, Lipari, 1985; A. RAFFA, La fine della Lipari medioevale. La guerra marittima turco-franco-spagnola del 1543-44 e la distruzione di Lipari ad opera di Barbarossa, in G.M. Bacci, M.A. Mastelloni (ed.), op. cit. (n. 11), p. 87-95.

${ }^{36}$ Archivio Segreto Vaticano (= ASV), Congr. Concilio, Relationes Diocesium, 456A, ff. 1v, 27r, 46r-47v, 54r, $103 r$.

37 Ibidem, 456B, f. 132r.

$3^{8}$ Ibidem, f. $146 r$.

${ }^{39}$ M. TABANELLI, Unica, alta et oblonga nave ad modum crucis extructa. Nuovi dati sulla cattedrale normanna di Lipari dalle visite ad limina Apostolorum dell'Archivio Segreto Vaticano, in In corso d'opera, 2. Ricerche dei dottorandi in Storia dell'arte della Sapienza, Roma, c.d.s.

${ }^{40}$ L. BERNABÒ BREA, Lipari, i vulcani, l'inferno e San Bartolomeo. Le isole Eolie dal Tardo Antico ai Normanni, in Archivio Storico Siracusano, 5, 1978/1979, p. 26-9o; ID., Il chiostro normanno di Lipari. La sua scoperta, il suo restauro, in U. Spigo, A. Raffa, M. Saija (ed.), Dal "constitutum" alle "controversie liparitane". Le chiavi di lettura della storia eoliana nell'ultimo millennio, Messina, 1998, p. 11-28; L. BERNABÔ BREA et alii, Il monastero normanno di Lipari e il suo chiostro. Ricerche e scavi (1954-1996), in Quaderni di Archeologia dell'Università di Messina, 2, 2001, p. 171-268.

${ }^{41}$ Ibidem, p. 183.
} 


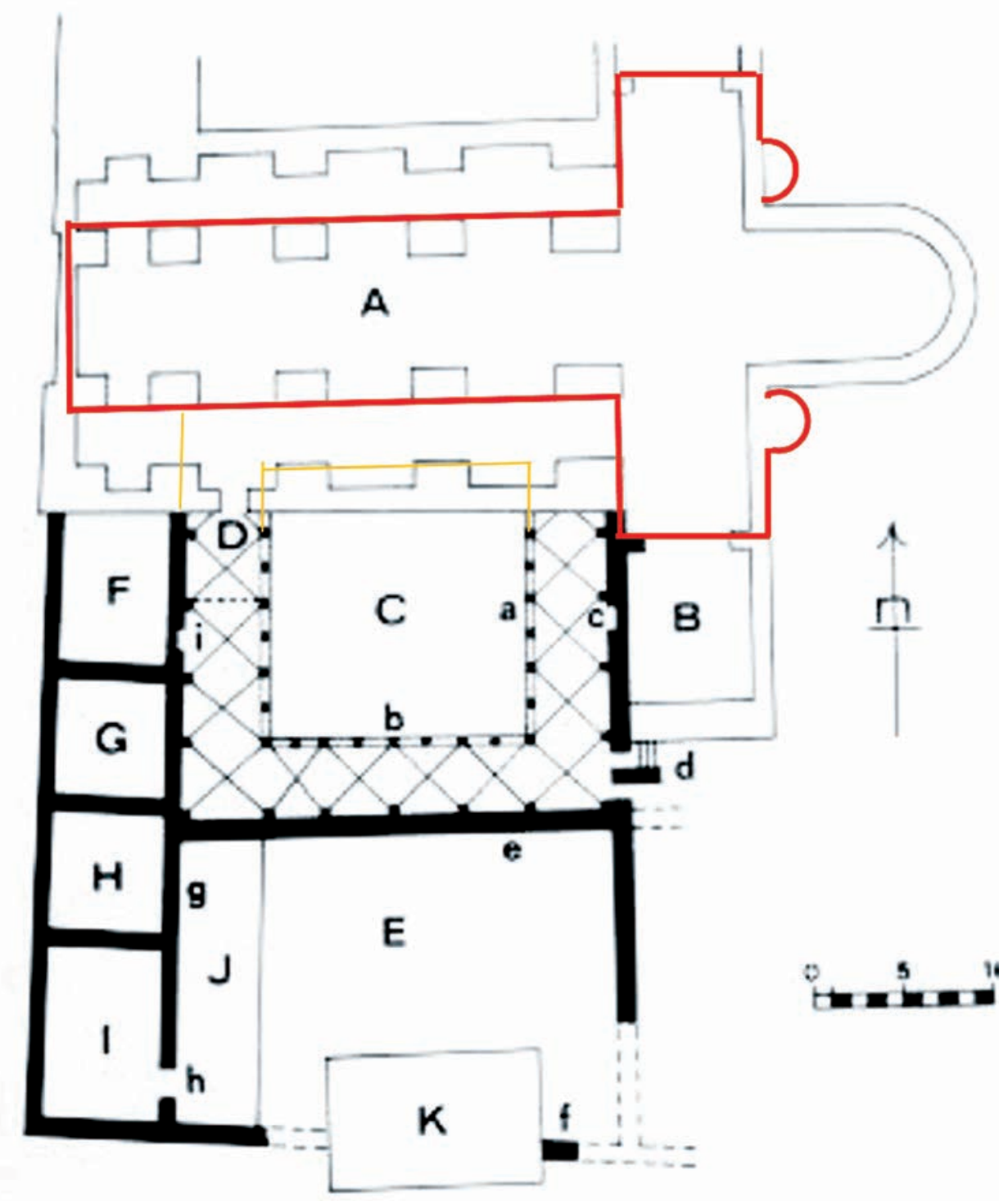

Fig. 1. Pianta del complesso cattedrale di Lipari con ipotesi restitutiva della chiesa originale. A: cattedrale; B: cappella del Santissimo Sacramento; C: chiostro; D: battistero; E: cortile epigrafico del Museo Archeologico; F, G, H, I: sale del Museo; K: padiglione epigrafico. Rielaborazione dell'Autrice da L. BERNABÒ BREA, Le Isole Eolie dal Tardo Antico ai Normanni, Ravenna, 1988. verso nord tale cortile è la stessa cui si appoggia sull'altro fronte la corsia meridionale del chiostro. Il giardino ha sostituito un'ulteriore ala a due piani del monastero, comunicante con il quadrato claustrale tramite almeno due porte. Nel livello superiore sopravvivono i resti di due monofore, la cui strombatura chiarisce come la sala che illuminavano si trovasse sul luogo del cortile epigrafico e non al di sopra del chiostro. Di questo fabbricato sono individuabili anche segmenti delle pareti laterali: l'orientale è il già visto proseguimento del vano scala, l'occidentale è reimpiegata come muro di spina tra le due file di sale del Museo. Il setto di fondo a sud è noto tramite alcuni sondaggi eseguiti presso il padiglione epigrafico che si affaccia sul giardino ${ }^{43}$.

Gli scavi condotti nel 1970 nelle sale al piano terreno della sezione preistorica del Museo hanno messo in luce una serie di piccoli ambienti costruiti al di sopra di uno strato con tracce di incendio, forse quello del $1544^{44}$. A un livello più profondo furono rintracciati i resti sia delle muraglie perimetrali est e ovest che dei setti divisori tra i diversi vani dell'ala monastica occidentale, affacciata sulla via principale dell'antica acropoli. Tali strutture furono reimpiegate come fondazioni al momento della ricostruzione del palazzo vescovile tra XVIII e XIX secolo, la cui fronte stradale ricalca quella del monastero normanno ${ }^{45}$.

La regolare disposizione dei corpi di fabbrica intorno a un cortile quadrilatero suggerisce che il chiostro, benché costruito in un secondo momento, fosse presente dall'origine a livello progettuale. Infatti nonostante la cesura ben leggibile con il transetto, gli ambienti conservati doppio arco aperto nella parete dell'ultima campata angolare SE, che dall'esterno, dove ora si trova uno scosceso giardino affacciato sul mare. Sono ancora conservate, benché tamponate, le porte d'accesso ai due vani sovrapposti, l'inferiore prospettante sulla campata mediana del braccio orientale del chiostro, l'altra sulla terrazza soprastante. In base a posizione e ampiezza si può ipotizzare che si trattasse della sala capitolare - al piano terreno - e del dormitorio dei monaci ${ }^{42}$.

Non è nota l'estensione verso sud del vano ospitante la scala, attualmente chiuso da un seriore muro in blocchetti, ma il perimetrale ovest prosegue per qualche metro nell'odierno cortile epigrafico del Museo. La parete che delimita non possono essere identificati in preesistenze di X o XI secolo, come invece è stato proposto ${ }^{46}$. Ci si trova di fronte a un complesso monastico di matura progettazione, non riconducibile certamente a un momento precedente all'impianto della comunità governata da Ambrogio.

\section{IL CHIOSTRO}

Il chiostro consta oggi di tre bracci: l'est e l'ovest mutili delle terminazioni verso la chiesa, il sud integro, per un totale di dodici campate complete e due dimezzate (fig. 2). Il perimetro misura $24,16 \mathrm{~m}$ per il lato sud e rispettivamente

\footnotetext{
${ }_{42}$ R. LEGLER, Der Kreuzgang. Ein Bautypus des Mittelalters, Frankfurt am Main, 1989, p. 207-212; R. ABEGG, Funktionen des Kreuzgangs im Mittelalter. Liturgie und Alltag, in Kunst und Architektur in der Schweiz, 48, 2, 1997, p. 18-20.

${ }^{43}$ L. BERNABÒ BREA, op. cit. (n. 40), p. 210.

${ }^{44}$ Ibidem, p. 210-211.

${ }^{45}$ Ibidem, p. 211-213.

${ }^{46}$ V. GIUSTOLISI, Della conquista di Lipari araba da parte della flotta pisana nell'anno 1035 e della presunta asportazione delle reliquie di San Bartolomeo apostolo, Palermo, 2010, p. 138-139.
} 


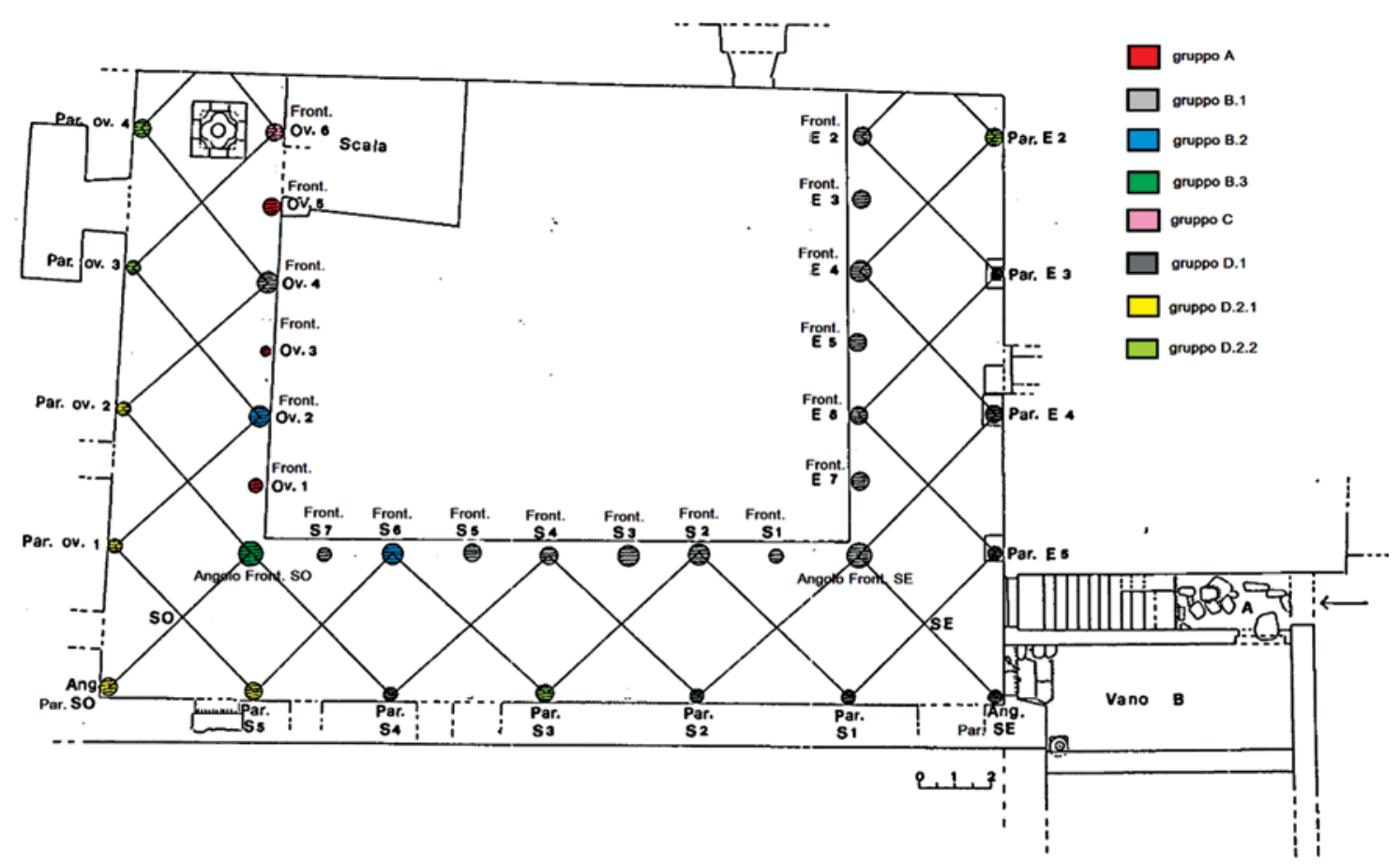

Fig. 2. Lipari, San Bartolomeo, pianta del chiostro con sigle di riferimento per la schedatura di colonne e capitelli. Rielaborazione dell'Autrice da L. BERNABÒ BREA-M. CAVALIER, Il monastero normanno di Lipari e il suo chiostro. Ricerche e scavi (1954-1996), in Quaderni di Archeologia dell'Università di Messina, 2, 2001, p. 171-268.

16,8 e 16,3 m per gli incompleti lati ovest e est. In base al passo medio delle campate, tuttavia leggermente irregolare, si può restituire una lunghezza originaria intorno ai $22,5 \mathrm{~m}$ per i due bracci mutili. L'impianto è lievemente trapezoidale per l'inclinazione della corsia occidentale, presumibilmente dettata dalla necessità di inserire il complesso tra l'antico decumano dell'acropoli e la scarpata verso il mare.

Due giri di tozze colonne, 36 in totale, sorreggono volte a crociera nervate (fig. 3-4). Il colonnato interno, unificato da un basso muretto che doveva ospitare dei sedili, ha passo dimezzato e conta quindi 21 sostegni, contro i 15 disposti a parete, collegati da archetti in sottili conci di pietra lavica ${ }^{47}$. Il peso delle coperture in scaglie litiche grava quindi nel fronte interno su una colonna ogni due, mentre lo scarico statico

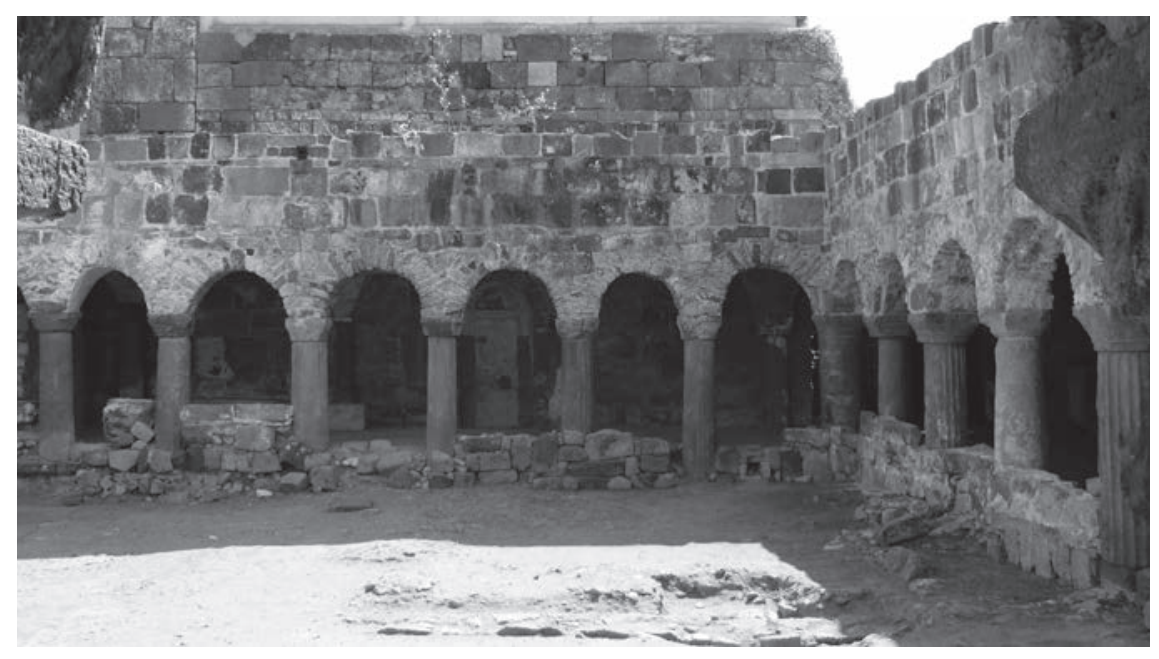

Fig. 3. Lipari, San Bartolomeo, chiostro, bracci est e sud visti da ovest (foto dell'Autrice). sulle muraglie perimetrali è garantito soltanto dall'incastro dei massicci capitelli a imposta, investendo il colonnato esterno di una mera valenza estetica. Le volte del chiostro non fanno corpo con la muratura perimetrale e pure la mancata armonizzazione delle arcate con gli ingressi degli ambienti monastici, i cui stipiti spesso risultano nascosti dalle colonne, indica come l'ambulacro appartenga a una fase edilizia successiva (fig. 4). Anche la tecnica muraria differisce da quella di chiesa e monastero, pur nell'omogeneità di materiali, e predilige elementi di piccolo formato disposti meno ordinatamente, forse da mascherare con un'intonacatura (fig. 3).
Rilevante è il reimpiego di fusti dorici in riodacite bruna locale, secondo Bernabò Brea cavati dai peristili di due differenti domus repubblicane ${ }^{48}$. Il primo gruppo (serie A), con diametro tra i 48 e $52 \mathrm{~cm}$, è collocato esclusivamente nel giro verso il cortile ${ }^{49}$. Colonne più esili, dal diametro di circa 42 cm (serie B), sono state invece prevalentemente addossate alle pareti perimetrali5o. I restanti sostegni furono realizzati al momento dell'erezione del chiostro da maestranze non particolarmenteabili, che ottennero prodotti poco omogenei e ancor meno regolari ${ }^{51}$. Agli angoli del peristilio interno si destinarono elementi molto più robusti degli altri, almeno da quanto si appura nei due esemplari superstiti (SE e SO).

47 L. BERNABÒ BREA, M. CAVALIER, op. cit. (n. 40), p. 182. Due dei sostegni interni sono di restauro. In origine le colonne dovevano essere 56: 24 lungo il perimetro esterno e 32 nel giro interno verso il giardino.

${ }^{48}$ Ibidem, p. 189-190.

${ }_{49}$ Front. E 2, 3, 4, 6, 7; Front. S 2, 4, 6; Front. O 2.

${ }^{50}$ Par. E 2; Front. E 5; Par. S 1, 2, 4; Front. S 5; Par. O 1, 2, 4; Front. O 5, 6.

${ }^{51}$ In latitandesite: Par. E 3. In riodacite: Par. E 4, 5, Angolo Par. SE; Par. S 3, 5; Front. S 1, 7 (restauro); Angolo Par. SO; Par. O 1, 3 ; Front. O 1. In pietra di San Calogero: Angolo Front. SE; Angolo Front. SO; Front. O 4. In granito grigio: Front. S 3. Una colonna in riodacite rinvenuta nei restauri è stata inserita 


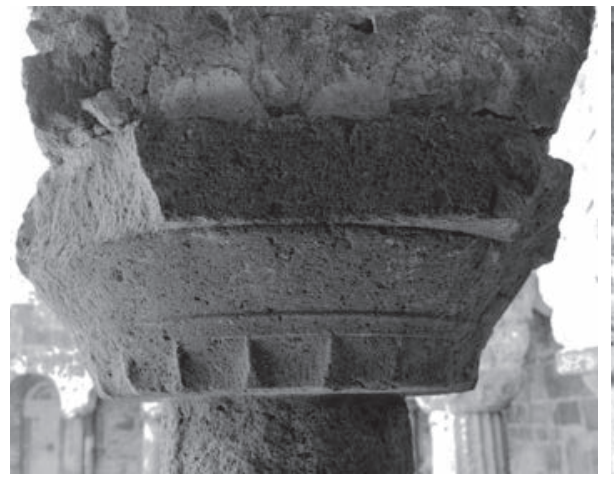

Fig. 5. Lipari, San Bartolomeo, chiostro, capitello del gruppo A (Front. O 3) (foto dell'Autrice).

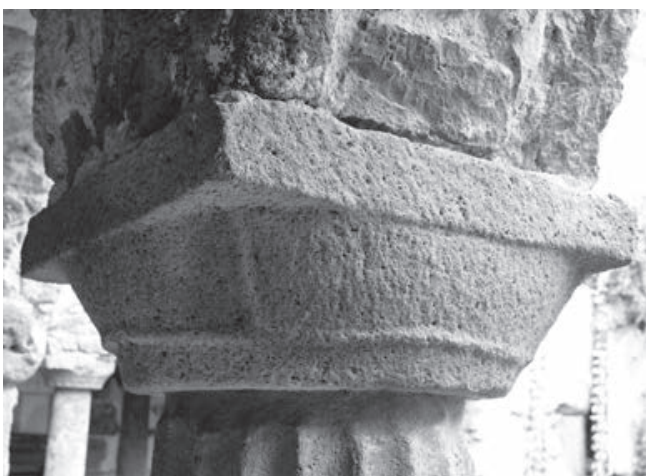

Fig. 6. Lipari, San Bartolomeo, chiostro, capitello del sottogruppo B1 (Front. S 5) (foto dell'Autrice).

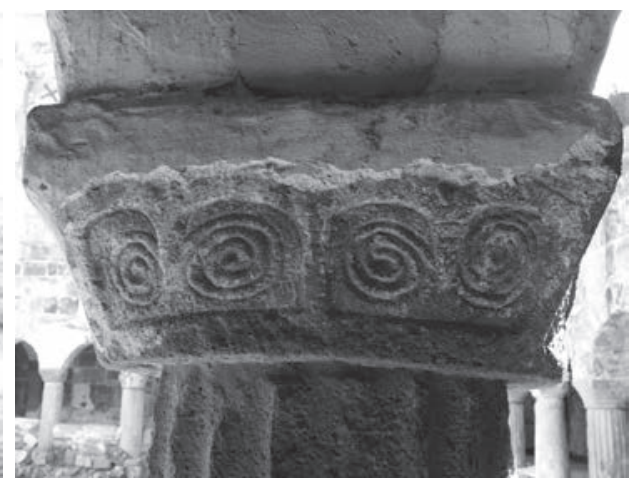

Fig. 7. Lipari, San Bartolomeo, chiostro, capitello del sottogruppo B.2 (Front. O 2) (foto dell'Autrice).

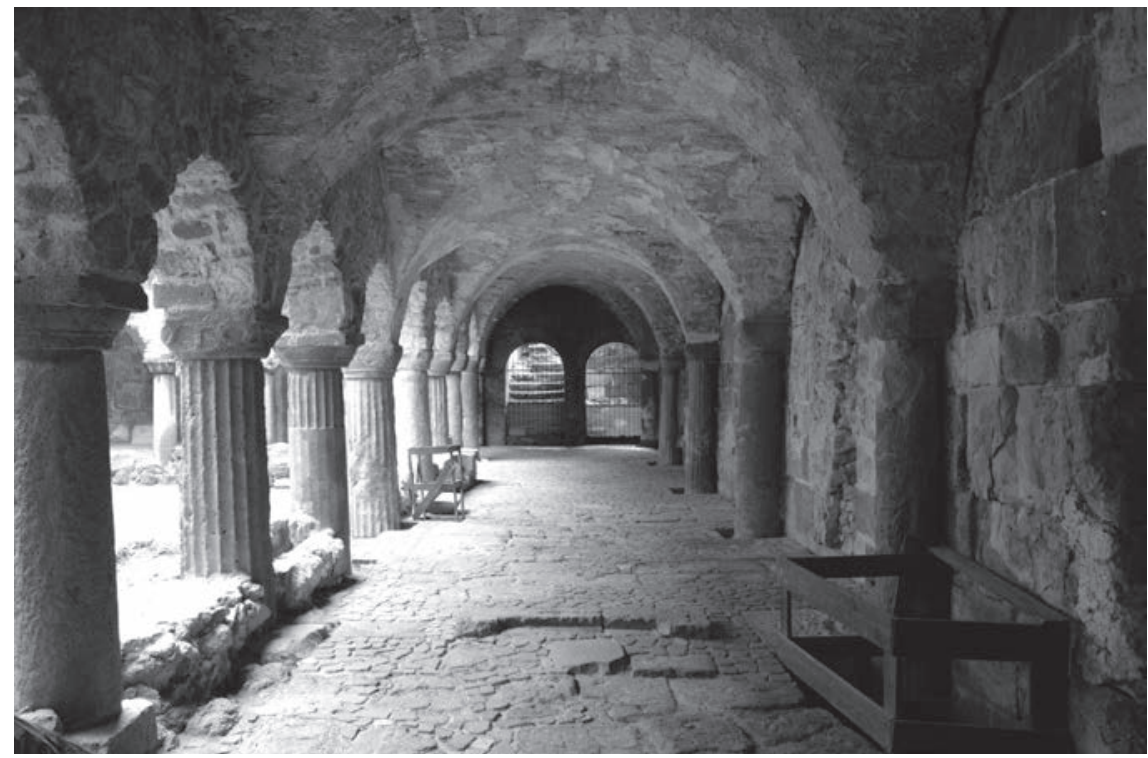

Fig. 4. Lipari, San Bartolomeo, chiostro, braccio sud (foto dell'Autrice).

Le pietre di costruzione provengono tutte da cave locali e sono di natura vulcanica: la riodacite bruna di Monte Sant'Angelo, la latitandesite del Monte Rosa e la cosiddetta pietra di San Calogero, una riodacite con venature violacee ${ }^{52}$. L'attività di estrazione era quindi ancora vitale in età normanna e la buona fattura dei conci sagomati delle aperture e degli archetti ciechi absidali della chiesa indica che fossero attive sul cantiere maestranze avvezze al taglio della pietra. La scelta di recuperare così tanto materiale di spoglio rispose quindi non tanto alla necessità di supplire alle mancanze degli operai disponibili, quanto all'opportunità di sveltire il cantiere e a considerazioni di ordine estetico. Sia le colonne che i capitelli dorici, infatti, furono collocati in posizione preminente nel peristilio, lasciando nell'ombra del perimetro esterno i nuovi fusti lisci.

Decisamente più scarso doveva essere il materiale scultoreo antico disponibile, dato che solo cinque sono i capitelli dorici rintracciabili, di cui uno trasformato in base (gruppo A)53 (fig. 5). A tale carenza supplirono gli scalpellini medievali, imitando liberamente i prototipi a disposizione. Le pietre utilizzate sono ancora una volta locali, con prevalenza della riodacite bruna, affiancata dalla latitandesite violacea. La maggioranza dei capitelli liberi può essere definita di tipo pseudo-dorico, seppur non si riscontri una perfetta omogeneità né di dimensioni né di lavorazione all'interno del gruppo (gruppo B). Caratteristiche comuni, desunte dai modelli antichi, sono la base circolare e l'abaco quadrato, ma l'echino può assumere un profilo più o meno tondeggiante e riportare in qualche caso una decorazione.

Una gran parte degli esemplari non ha alcun ornamento, a esclusione di pronunciati cordoli a profilo torico collocati subito al di sopra della base e più raramente lungo gli angoli (sottogruppo B.1) ${ }^{54}$ (fig. 6). Il livello qualitativo è nel complesso piuttosto scadente e in molti manufatti si è rinunciato alla prevista ortogonalità delle facce, ottenendo oggetti davvero rudimentali. Alcuni emergono tuttavia dalla massa quali frutti di mani più esperte, capaci di ottenere una corretta stereometria e più precise cornici55.

I pochi esemplari decorati si concedono semplici motivi geometrici o vegetali. Due capitelli pseudodorici in latitandesite hanno ciascuna faccia dell'echino scandita da brevi archeggiature ospitanti vortici (sottogruppo B.2, fig. 7). Sono disposti simmetricamente rispetto alla colonna angolare di SO (in posizione Front. O 2 e Front. S 6) ed entrambi si sovrappongono a fusti di reimpiego scanalati. E forse proprio a ciò si deve la scelta di apporvi un'ornamentazione ad archet$\mathrm{ti}$, che crea l'effetto di prolungare e concludere le scanalature. Tra i due, quello della corsia ovest è sicuramente il meglio riuscito: presenta una più regolare squadratura del blocco e anche il tracciato dei decori è molto più sicuro in confronto al "gemello" meridionale. Il capitello della colonna angolare SO reca foglioline appena rilevate in corrispondenza degli

$\overline{\text { in Front. } \mathrm{S} 7 \text { in sostituzione di un sostegno perduto, mentre in Front. } \mathrm{O}} 3$ è riutilizzata una colonna scanalata esile rinvenuta anni prima nel cortile del sacrestano (Ibidem, p. 190, 195).

${ }^{2}$ Ibidem, p. 190.

53 Ibidem, p. 189. Due capitelli sormontano le colonne Front. O 1, 3 (posizionato nel restauro, forse proveniente dal braccio nord), mentre un terzo di uguali dimensioni è la base del fusto Par. S 3. Sono compatibili con le colonne della serie A. Un quarto capitello, più piccolo, è stato rinvenuto nella demolizione dei tramezzi dell'ambulacro sud. Bernabò Brea ne ipotizza la provenienza dal braccio nord. Anche il capitello della colonna Front. O 5 è dorico di reimpiego ma mutilo.

${ }^{54}$ Hanno il cordolo solo alla base i capitelli: Front. O 4; Front. S 2, 3, 4, 7; Angolo Front. SE; Front. E 3, 4, 6, 7. Presentano anche nervature angolari: Front. $\mathrm{S}_{1}$, 5; Front. E 2, 5 .

55 In particolare i capitelli nelle posizioni: Front. O 4; Front. S 7; Front. E 2; Angolo Front. SE. 


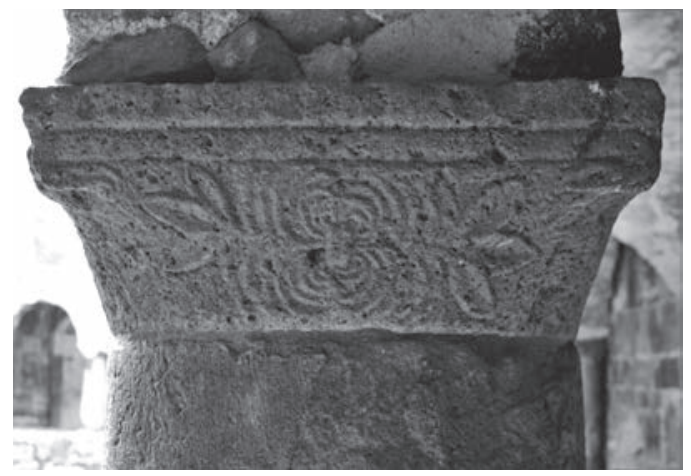

Fig. 8. Lipari, San Bartolomeo, chiostro, capitello del sottogruppo B.3 (Angolo Front. SO) (foto dell'Autrice).

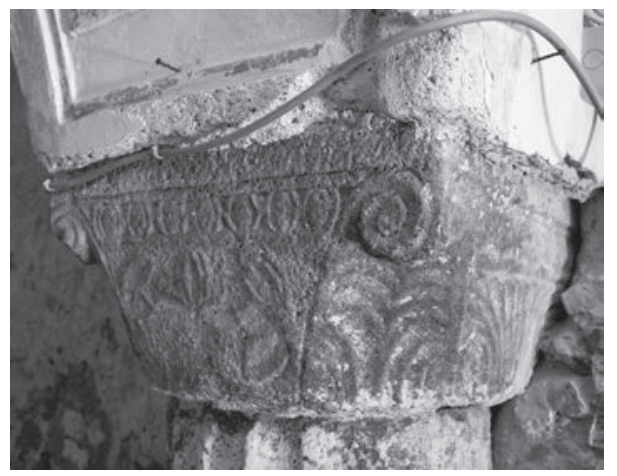

Fig. 9. Lipari, San Bartolomeo, chiostro, capitello del gruppo C (Front. O 6). Foto dell'Autrice.

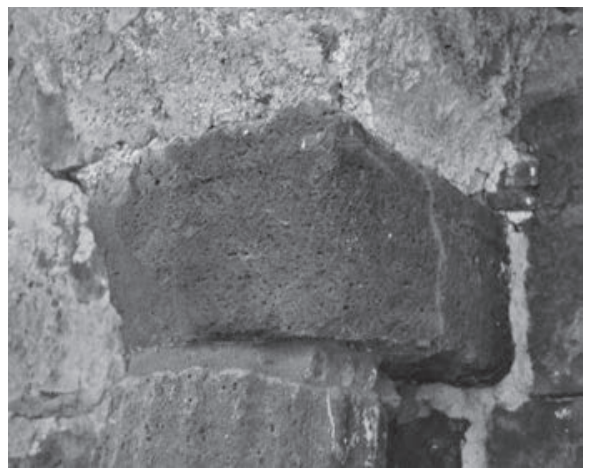

Fig. 10. Lipari, San Bartolomeo, chiostro, imposta del gruppo D.1 (foto dell'Autrice).

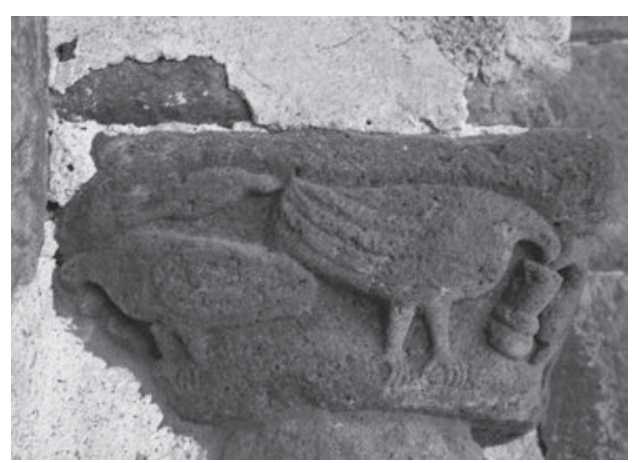

Fig. 11. Lipari, San Bartolomeo, chiostro, capitello del sottogruppo D.2.1 (Angolo Par. SO) (foto dell'Autrice).

spigoli e solo sulla faccia occidentale un fiore a lobi concentrici circondato da due ciuffi di foglie, il tutto reso attraverso una delicata incisione (sottogruppo B.3, fig. 8). Molto più ricco ma con una simile lavorazione a bassissimo rilievo è il primo capitello che si incontra sulla sinistra accedendo al chiostro dal moderno ingresso (Front. O 6, fig. 9). Questo rielabora in forme semplificate il modello del capitello composito romano, forse sulla base di un concreto prototipo locale, associando solide volute angolari a un solo giro di ampie foglie d'acanto, cui si

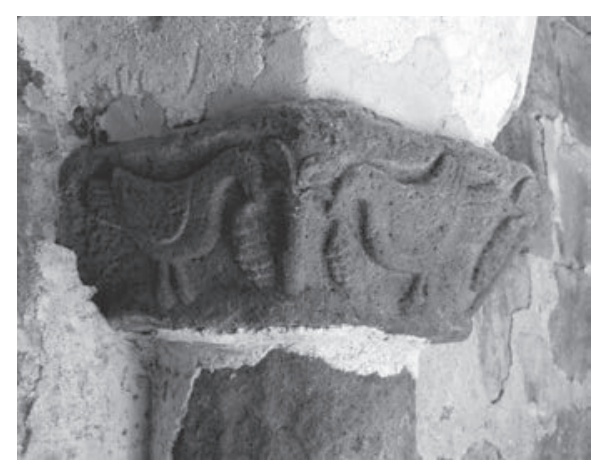

Fig. 12. Lipari, San Bartolomeo, chiostro, capitello del sottogruppo D.2.1 (Par. O 2) (foto dell'Autrice).

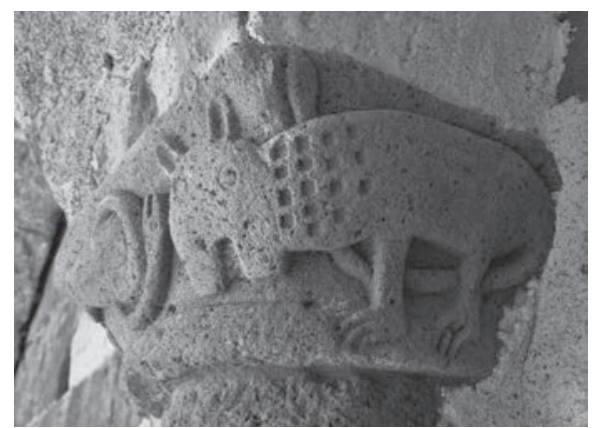

Fig. 14. Lipari, San Bartolomeo, chiostro, capitello del sottogruppo D.2.1 (Par. S 5) (foto dell'Autrice).

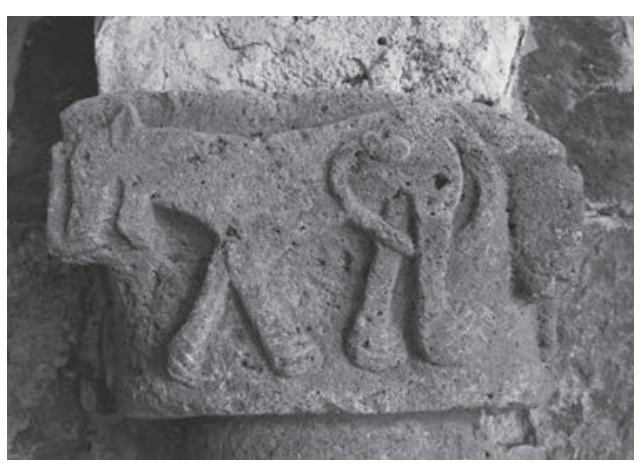

Fig. 13. Lipari, San Bartolomeo, chiostro, capitello del sottogruppo D.2.1 (Par. O 1) (foto dell'Autrice).

un'intera faccia e ogni palma o cantaro un angolo, si è ottenuta per ogni imposta una sola coppia simmetricamente affrontata, relegando il terzo uccello a compiere solitariamente la stesa azione sull'ultimo lato disponibile. Negli altri due esemplari figurati sono di ben più ardua identificazione gli animali rappresentati. L'ultimo del braccio occidentale (Par. O 1, fig. 13) raffigura tre bestie con lunghe code arrotolate tra le zampe, forse terminanti con artigli, musi allungati aperti a scoprire $\mathrm{i}$ denti e orecchie appuntite. A prima vista sembrano assimilabili a lupi, ma forse è sovrappone un'alta fascia a ovuli (gruppo C).

Le imposte del colonnato addossato alle murature perimetrali del chiostro sono invece state integralmente prodotte ex novo (gruppo D). Si tratta in maggioranza di semplicissimi blocchi rettangolari, di squadratura più o meno precisa, parzialmente incassati nella parete per trasmetterle il peso delle crociere (sottogruppo D.1, fig. 10). Alcuni elementi, concentrati lungo il segmento occidentale del perimetro e presso l'interruzione del braccio est, esibiscono invece decori animalistici o vegetali (sottogruppo D.2) ${ }^{56}$.

I primi dispongono tendenzialmente le figure di animali, rese a piatte sagome con dettagli incisi e ben rilevate dal fondo, isolatamente su ciascun lato, cercando faticosamente in qualche occasione di sfruttare gli angoli per porle in comunicazione (sottogruppo D.2.1). E il caso delle due imposte con colombe, raffigurate nell'atto di abbeverarsi a un cantaro (Angolo Par. SO, fig. 11) o di becchettare dei datteri (Par. O 2, fig. 12). Dato che ogni volatile occupa più verosimile che si tratti di leonesse in considerazione della coda liscia terminante in un ciuffo di peli. Si dispongono secondo lo schema evidenziato per le colombe, ossia in una coppia a teste affrontate e un individuo autonomo. La prima imposta della corsia sud (Par. S 5, fig. 14) varia questa consuetudine compositiva, associando animali diversi in lotta tra loro. Un grande pesce contende la preda a un altro animale acquatico, allo stesso tempo stretto dalle spire di un serpente, la cui testa si accosta a quella di una tozza bestia con capo e orecchie arrotondate, fauci spalancate, lunga coda esile con fiocco finale e la parte superiore del torace segnata da intagli quadrati. Questo particolare costituisce forse la traduzione su pietra di un motivo presente in un modello grafico e potrebbe indicare la criniera di un leone.

Passando agli esemplari con ornati vegetali (sottogruppo D.2.2), ci si trova ancora di fronte a una coppia di "gemelli" con piccole variazioni, collocata ai due opposti del quadrato claustrale ma non in perfetto allineamento (Par. O 3 e Par. E

${ }_{56}$ Nelle posizioni Par. O 1, 2, 3, 4, Angolo Par. SO; Par. S 5; Par. E 2. 


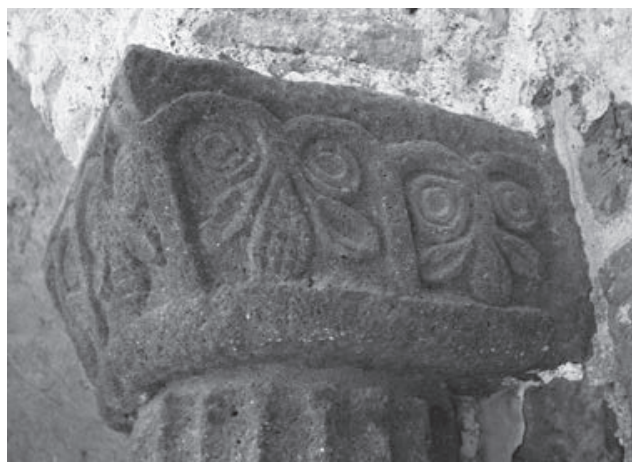

Fig. 15. Lipari, San Bartolomeo, chiostro, capitello del sottogruppo D.2.2 (Par. E 2) (foto dell'Autrice).

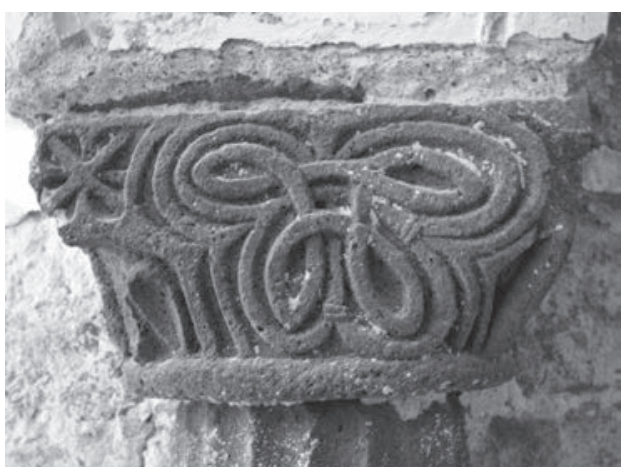

Fig. 16. Lipari, San Bartolomeo, chiostro, capitello del sottogruppo D.2.2 (Par. O 4) (foto dell'Autrice).

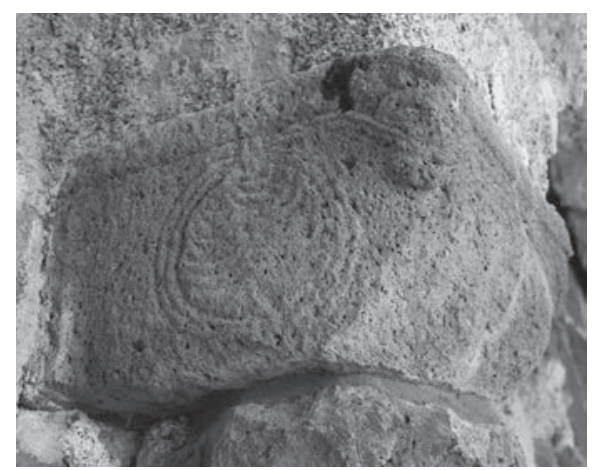

Fig. 17. Lipari, San Bartolomeo, chiostro, capitello del sottogruppo D.1 (Par. S 4) (foto dell'Autrice).
2, fig. 15). Il motivo decorativo è costituito dalla ripetizione lineare di penduli gruppi di tre foglie carnose strette da due girali desinenti in elementi verticali. L'effetto d'insieme è simile a quello dei capitelli pseudodorici con archetti e vortici (sottogruppo B.2, fig. 7), seppur con impaginazione più rigorosa e intagli molto netti e approfonditi. L'imposta orientale si differenzia per la lavorazione della foglia centrale, trasformata in grappolo o pigna. L'imposta Par. O 4, di fattura molto vicina alla coppia appena esaminata, attinge a un repertorio prettamente aniconico e presenta sui lati brevi delle profonde eallungate scanalature, mentre la faccia principale è occupata da un nodo a quattro asole arrotondate ripetuto in tre giri concentrici (fig. 16). In ultimo, l'elemento Par. S 4, decorato a leggera incisione con volute di nastri fuoriuscenti da maschere mostruose angolari (fig. 17), si avvicina molto dal punto di vista esecutivo ai già ricordati capitelli Front. O 6 e soprattutto Angolo Front. SO.

In ultimo va spesa qualche parola in merito al sostegno a parete in posizione Par. E 3, composto da una colonnina in latitandesite violacea di anomale proporzioni con semplice imposta quadrangolare e alta base con elementi globulari negli angoli, simili a crochet. Krönig osservava a ragione che tale basamento deve risalire per lo meno al XII secolo inoltrato, trascinando di conseguenza l'intero cantiere in quell'epoca ${ }^{57}$. Lo studioso tedesco non notava tuttavia che il tratto di muratura in cui si innesta il capitello e cui semplicemente si appoggiano colonna e base costituisce un rappezzo in blocchetti più minuti e disordinati dell'apparecchio in grandi conci di prima fase e che quindi l'intero sostegno ha quasi di certo rimpiazzato un elemento danneggiato.

A dispetto dell'altalenante livello qualitativo di capitelli e imposte e dell'assenza di corrispondenza dimensionale tra questi e i fusti, sia di reimpiego che di nuova produzione, emerge la volontà di ottenere un complessivo effetto di omogeneità giocando sulla disposizione dei manufatti disponibili. Al giro interno di colonne furono infatti destinati esclusivamente capitelli dorici di reimpiego e gli esemplari pseudodorici su di essi modellati. Nella corsia occidentale si nota l'accortezza di alternare i pezzi antichi a imitazioni particolarmente curate. La stessa ala ospita anche la quasi totalità delle imposte decorate, a eccezione di quella in posizione Par. E 2. Questa si trova infatti nell'immediata pros- simità dell'interruzione determinata dall'ampliamento della navata e si affronta a un capitello pseudodorico di fattura più elevata rispetto agli altri del braccio est. Ciò induce a ipotizzare che anche nel perduto lato settentrionale del chiostro fossero alloggiati elementi antichi e figurati e che quindi essi caratterizzassero circa due terzi del complesso, allungandosi dall'angolo sudovest a quello nordest. Non è possibile tuttavia trovare piena conferma a questa ricostruzione, dato che nei lavori di liberazione sono riemersi tra le macerie di riempimento solo due capitelli dorici, presumibilmente in origine allocati nel braccio perduto ${ }^{5}$. La valorizzazione decorativa della corsia aderente all'edificio ecclesiastico è d'altra parte fenomeno diffuso a livello europeo e spesso si estende allo spazio antistante la sala capitolare ${ }^{59}$. A Lipari sembra invece essere stato privilegiato il braccio opposto al fabbricato di capitolo e dormitorio, la cui fronte al contrario vede esclusivamente imposte e capitelli privi di decorazione. L'unico superstite capitello ornato sul lato est si posiziona proprio in corrispondenza del termine del transetto, subito prima dell'avvio della sala capitolare.

Nelle sopravvissute porzioni dei corridoi est e sud sono impiegati manufatti molto più dozzinali, che imitano con scarso successo gli esemplari migliori degli altri due lati. Si può supporre che ciò sia dovuto a una scelta strategica, mirata a isolare i prodotti meno soddisfacenti nel settore più lontano dalla chiesa, o all'abbandono del cantiere da parte delle maestranze più abili mentre l'impresa era ancora incompiuta.

L'ipotesi di Krönig di spostare verso la fine del XII secolo gli ambulacri di Lipari, curiosamente motivata da una presunta «mancanza di presupposti per un'architettura monumentale già nella prima generazione dei conquistatori $»^{60} \mathrm{che}$ ignora l'impressionante serie di fabbriche avviate tra i tardi anni cinquanta e gli anni novanta dagli Altavilla, non èa mio avviso più sostenibile. Da un lato la confezione complessiva con doppio giro di colonne e volte a crociera non accenna in alcun modo ai supposti prototipi di Cefalù e Monreale, dall'altro la scultura architettonica non sembra suscettibile di una datazione così bassa. E questo non tanto per la scarsa credibilità in termini di mimesis delle figure animali e degli ornati vegetali - perché scalpellini di poca abilità non sono mancati in tutte le epoche - quanto per la composizione

\footnotetext{
57 W. KRÖNIG, Sul complesso architettonico normanno contiguo alla Cattedrale di Lipari, in Archivio Storico Siracusano, 5, 1978/1979, p. 91-99; ID., Sul complesso architettonico normanno contiguo alla Cattedrale di Lipari, in L. BERNABŌ BREA, op. cit. (n. 5), p. 145-162.

${ }^{8}$ V. supra, n. 53 .

${ }^{9}$ P. KLEIN, Zur Einführung: Der mittelalterliche Kreuzgang, in P. Klein (ed.), Der mittelalterliche Kreuzgang. Architektur, Funktion und Programm, Regensburg, 2004, p. 15-16.

${ }^{60}$ W. KRÖNIG, Sul complesso architettonico, op. cit. (n. 57).
} 


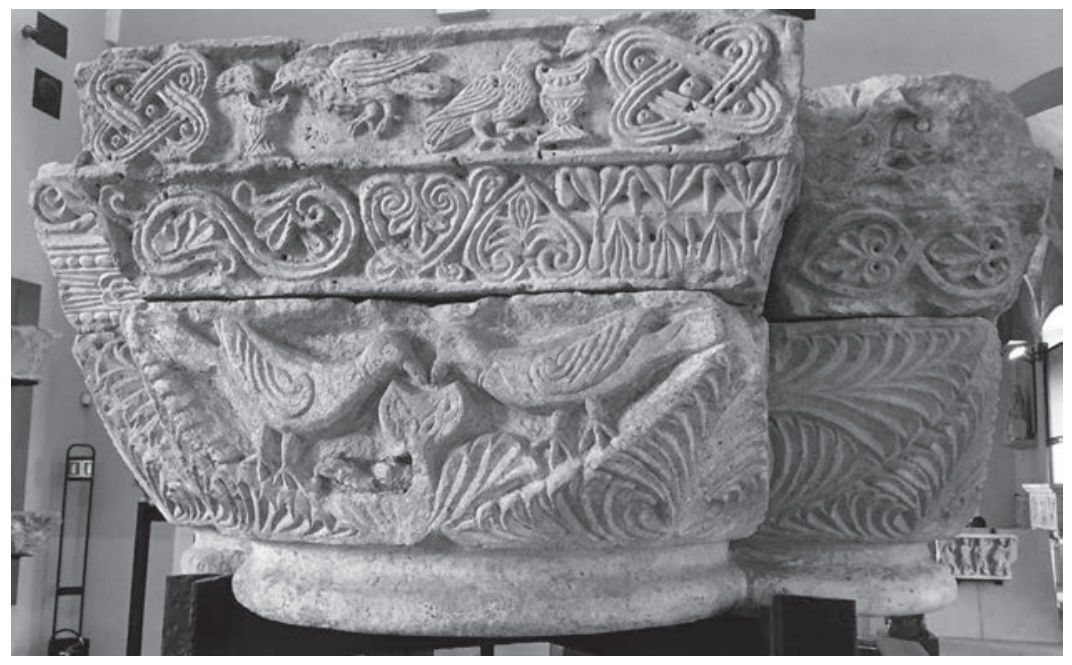

Fig. 18. Milano, Civiche Raccolte d'Arte Antica, capitello da Santa Maria d'Aurona (foto dell'Autrice).

del programma decorativo. Mancano infatti totalmente le scene narrative, che soprattutto da metà XII proliferano sui capitelli dei chiostri italiani ${ }^{61}$, e come si è visto la figurazione si riduce a sagome di animali disposte paratatticamente. Lo stesso repertorio geometrico e vegetale sembra appartenere alla tradizione protoromanica di XI secolo, a sua volta debitrice dell'ornato altomedievale: intrecci spigolosi, vortici e penduli elementi vegetali racchiusi da girali. Un notevole compendio dei principali motivi decorativi delle imposte di Lipari è offerto da un capitello di pilastro proveniente dalla fase romanica di Santa Maria d'Aurona a Milano (XI-XII sec., fig. 18$)^{62}$.

La conservatività delle formule decorative non autorizza tuttavia, come invece si è proposto, a ritenere che si tratti di pezzi prodotti durante i secoli della dominazione islamica e reimpiegati nella struttura normanna ${ }^{63}$, sia per considerazioni stilistiche che per il semplice fatto che la lavorazione tiene conto del necessario incastro a muro. Pur ammettendo un certo attardamento delle maestranze attive a Lipari, se il cantiere fosse stato intrapreso sulla scia delle iniziative reali di Cefalù e Monreale almeno qualche tentativo, seppur molto naif, di replicarne le novità avrebbe dovuto palesarsi. La bottega doveva comporsi di due o tre maestri di maggiore abilità, di cui uno specializzato in decori disegnati da linee a

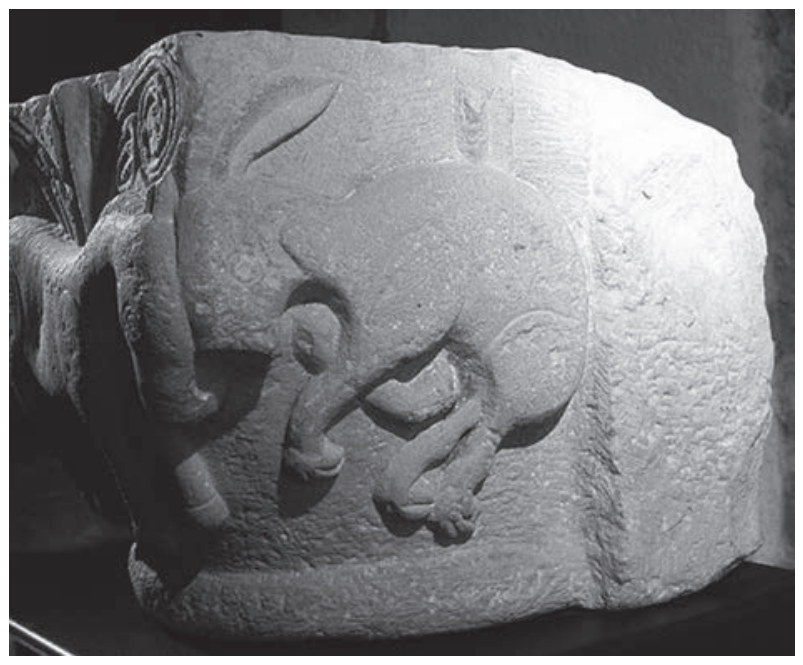

Fig. 19. Ventimiglia, cattedrale, capitello erratico della cripta (foto dell'Autrice).

leggerissimo rilievo e gli altri più propensi a lavorare per intagli netti e forme ampie e piatte. A questi artefici si affiancò un certo numero di tagliapietre improvvisatisi scultori, che con molte difficoltà tentarono di replicare i modelli proposti senza avventurarsi nell'ornamentazione.

La provenienza dei "capobottega" di Lipari è di davvero ardua individuazione. Se ne può escludere la formazione meridionale, data l'assenza di riscontri con le poche tracce superstiti in Sicilia della scultura d'età prenormanna ${ }^{64}$, così come non si evidenziano tangenze con le maestranze attive negli ultimi decenni dell'XI secolo nei cantieri campani e lucani ${ }^{65}$. Non vi sono nemmeno affinità con la produzione dell'officina dello scultore Gandolfo, attiva nel secondo quarto del XII secolo ${ }^{66}$, o con i rari capitelli realizzati ad hoc per la cattedrale di Catania ${ }^{67}$. Poco convincente è il già proposto accostamento ai più maturi frammenti di scultura architettonica provenienti da Santa Lucia di Mendola ${ }^{68}$.

Sembra praticabile allora l'ipotesi dell'appartenenza dello scultore alla composita colonia centrosettentrionale impiantatasi nelle Eolie a partire dalla fine del Mille. E se piatte sagome di animali più o meno mostruosi popolano gli edifici delle regioni centrosettentrionali tra XI e XII secolo ${ }^{69}$ (fig. 19), è soprattutto dall'area centroitaliana che arrivano le testimonianze protoromaniche di incisioni ornamentali

\footnotetext{
$\overline{{ }^{6}}$ Si pensi, oltre che a Cefalù e Monreale, a Sant'Orso di Aosta e Santa Sofia di Benevento.

${ }^{62}$ Ora conservato nelle Civiche Raccolte d'arte antica di Milano. L'intero complesso di frammenti scultorei proveniente da Santa Maria d'Aurona era stato inizialmente ritenuto di VIII secolo, ma ora le imposte da pilastro sono state agganciate all'intervento di aggiunta delle volte tra XI e XII secolo: cfr. oltre al saggio nel presente volume, anche R. CASSANELLI, Santa Maria d'Aurona: la fase romanica, in R. Cassanelli, P. Piva (ed.), Lombardia romanica, 2. Paesaggi monumentali, Milano, 2011, p. 42-44.

${ }_{3}^{6}$ E. MOLTENI, I capitelli del chiostro di Lipari. Scultura normanna e di reimpiego nel complesso della cattedrale, in V. Giustolisi (ed.), Alla ricerca di Lipari bizantina, Palermo, 2001, p. 37-58.

${ }_{4}^{64}$ G. AGNELLO, Sculture bizantine della Sicilia, in Siculorum Gymnasium, 5, 1952, p. 76-91; 6, 1953, p. 222-235; 7, 1954, p. 104-117; 10, 1957, p. 101-122.

${ }_{5}^{6} \mathrm{Mi}$ riferisco in particolare alle cattedrali di Aversa, Acerenza e Salerno e alla Santissima Trinità di Venosa. Per una panoramica sulla prima scultura normanna: D. GLASS, Romanesque sculpture in Campania. Patrons, Programs, and Style, University Park (Pennsylvania), 1991, p. 35-64; P. BELLI D’ELIA, La produzione scultorea nel Sud della Penisola, in M. D’Onofrio (ed.), I Normanni: popolo d'Europa 1030-120o, Venezia, 1994, p. $229-236$.

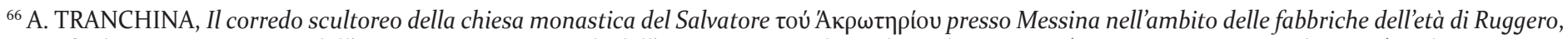
in Dialoghi con Bisanzio, Atti dell'VIII congresso nazionale dell'Associazione Italiana di Studi Bizantini (Ravenna, 22-25 settembre 2015), c.d.s.

${ }^{67}$ F. GANDOLFO, Le cattedrali siciliane, in A.C. Quintavalle (ed.), L'Europa delle cattedrali, Atti del convegno (Parma, 19-23 settembre 2006), Milano, 2007, p. 203.

${ }^{68}$ G. AGNELLO, Le sculture normanne di S. Lucia di Mèndola, in Bollettino d'arte del Ministero della Pubblica Istruzione, 21, 1927/1928, p. 586-595; S. L. AGNELLO, Scavi e scoperte a S. Lucia di Mèndola, in Atti del I Congresso nazionale di Archeologia cristiana, Siracusa, 19-24 settembre 1950, Roma, 1951, p. $49-58$.

${ }^{69}$ Tra i molti esempi possibili, si citano la lastra di sarcofago del Museo Sant'Agostino a Genova [tardo XI secolo: I.M. BotTo (ed.), Museo di Sant’Agostino, Genova, s.a., p. 52] edei capitelli di Sant'Ambrogioa Milano [prima metàXII secolo:A. SUMMA, La scultura decorativa medioevale nella basilica di S. Ambrogio, in M.L. Gatti Perer (ed.), La basilica di S. Ambrogio: il tempio ininterrotto, 2, Milano, 1995, p. 389-417; R. CASSANELLI, La basilica di Sant'Ambrogio a Milano, in R. Cassanelli,
} 
a vortice ${ }^{70}$. L'assenza di eloquenti peculiarità stilistiche nelle sculture del chiostro, unita all'omogeneità del repertorio ornamentale di questi secoli in vaste aree macroregionali, ostacolano purtroppo ogni tentativo di precisare l'origine del maestro di Lipari. Credo tuttavia sia possibile circoscriverne l'attività entro i primi decenni del XII secolo, senza dover forzatamente legare l'avvio del cantiere alla creazione della diocesi nel 1131, dato che l'affiancamento della funzione vescovile a quella monastica non introduce necessità funzionali tali da motivare la costruzione di una simile struttura, né, nel caso specifico, ha comportato un aumento delle risorse patrimoniali della comunità, il cui accrescimento si era anzi arrestato nei primi anni Venti.

La scelta del chiostro a tale altezza cronologica sembra essere un antefatto nel Meridione normanno. Non sappiamo nulla purtroppo sull'assetto degli ambienti monastici calabresi di Santa Maria di Sant'Eufemia, Santa Maria e Dodici Apostoli di Bagnara, Santa Maria della Torre a Serra San Bruno, mentre le piante secentesche di Mileto sembrano indicare la presenza di un chiostro a sud della chiesa ${ }^{71}$, da cui potrebbero però provenire i capitelli a stampella ora al Museo Statale, non certo riferibili alla fondazione degli anni sessanta del Mille ${ }^{72}$. In definitiva i più antichi chiostri noti con certezza nel Mezzogiorno italiano, ad eccezione del singolare caso di Cava dei Tirreni ${ }^{73}$, si collocano dopo la metà del XII secolo, come Cefalù ${ }^{74}$ e Santa Sofia di Benevento $^{75}$. Arduo è individuare la causa dell'impianto di tale moderna soluzione a Lipari. Si potrebbe forse pensare a una scelta dettata dalla provenienza dell'abate e di parte della prima comunità, dato che nel Nord Italia, tendenzialmente più rapido nella recezione delle tendenze architettoniche europee, l'apparizione dei primi chiostri si colloca per lo meno nei primi decenni del secolo ${ }^{76}$. Isolata - e rara anche a livello europeo - è pure l'adozione di una copertura a crociera per le corsie $\mathrm{e}^{77}$, che può ragionevolmente essere stata dettata dalla scarsità di legname idoneo nell'arcipelago. Le volte esibiscono elementi di relativo arcaismo come le nette nervature, un leggero andamento cupoliforme e una certa irregolarità di imposta, ma non necessitano di archi formeret e trasversali, il che ben si sposa con una datazione nei primi decenni del XII secolo.

Alla luce di queste riflessioni, appare evidente come il chiostro di San Bartolomeo rappresenti uno dei più emblematici episodi della strategia normanna di "latinizzazione" religiosa e culturale del Meridione, che si è espressa con maggiore forza laddove le esigenze politiche lo suggerivano e che ha finito per prevalere nel corso del XII secolo, una volta terminata l'utilità mediatrice nei confronti delle popolazioni autoctone del monachesimo greco.

P. Piva (ed.), Lombardia romanica, 1. I grandi cantieri, Milano, 2010, p. 125-146], il capitello erratico della cripta della cattedrale di Ventimiglia [1050-1080 ca.: F. CERVINI, Liguria romanica, Milano, 2002 (Patrimonio artistico italiano), p. 35-45], i capitelli del battistero di Arsago Seprio [metà XII secolo: L.C. SCHIAVI, Il complesso di Arsago Seprio, in R. Cassanelli, P. Piva (ed.), op. cit. (n. 62), p. 191-20o] e quelli dei pilastri in controfacciata di San Pietro a Gropina presso Loro Ciuffenna (metà XII secolo: F. GANDOLFO, San Pietro a Gropina, in W. ANGELELLI, F. GANDOLFO, F. POMARICI, La scultura delle Pievi. Capitelli medievali in Casentino e Valdarno, Roma, 2003, p. 25-53).

$7^{70}$ Ad esempio i capitelli della cripta del duomo di Fiesole, provenienti dalla chiesa iniziata nel 1028 e reimpiegati nell'edificio attuale [G. TIGLER, Toscana romanica, Milano, 2006 (Patrimonio Artistico Italiano), p. 167-169]; quelli della cripta di San Pietro a Leopoli-Cencelle [fine XI-inizio XII secolo: P.F. PISTILLI, La chiesa di San Pietro, in L. Ermini Pani, M. C. Somma, F. R. Stasolla (ed.), Forma e vita di una città medievale: Leopoli-Cencelle, Spoleto, 2014, p. 31-33], della cripta Santa Maria di Valfucina presso San Severino Marche [seconda metà XI secolo: C. CERIONI, Abbazia di Santa Maria di Valfucina (San Severino Marche), in P. Piva, C. Cerioni (ed.), Il Romanico nelle Marche, Milano, 2012, p. 149-150] e di quella di Santo Stefano a Lenno [seconda metà XI secolo: O. ZASTROW, Scultura carolingia e romanica nel comasco, Como, 1981, p. 120-121; M. ROSSI, La pieve di Lenno e altre questioni lariane, in A. Segagni Malacart, L. C. Schiavi (ed.), Architettura dell'XI secolo nell'Italia del Nord. Storiografia e nuove ricerche, Atti del convegno (Pavia, 8-10 aprile 2010), Pisa, 2013, p. 127-133].

${ }^{71}$ Pianta del 1581, Archivio del Collegio Greco di Roma, v. 83, f. A, pubblicata da G. OCCHIATO, La SS. Trinità di Mileto e l'architettura normanna meridionale, Catanzaro, 1977 .

${ }^{72}$ Nonostante l'opinione di M. P. DI DARIO GUIDA, La cultura artistica, in A. Placanica (ed.), Storia della Calabria medievale, 2, Roma, 1999, p. 172-173 e R. CAPUTO, Il Museo Statale di Mileto, Soveria Mannelli, 2004, p. 34-35.

73 G. PANE, La "Crypta cava" e la fabbrica antica, in G. Fiengo (ed.), La Badia di Cava, 1, s.l., 1985, p. 133-147.

${ }^{74}$ F. GANDOLFO, Il chiostro di Monreale, in M. D’Onofrio (ed.), op. cit. (n. 65), p. 237-243; C. VALENZIANO, Introduzione al Chiostro canonicale di Cefalù, Cefalù, 2008 .

75 F. GANDOLFO, La scultura normanno-sveva in Campania, Roma-Bari, 1999, p. 41-47; H. GIESS, The sculpture of the cloister of Santa Sofia in Benevento, in The Art Bulletin, 41, 1959, 3, p. 249-256.

${ }^{76}$ Per un riepilogo sulla comparsa dei chiostri in Italia settentrionale: C. TOSCO, L'architecture des cloîtres dans l'Italie du Nord (XIe-XII siècles), in Les Cahiers de Saint-Michel de Cuxa, 46, 2015, p. 61-76. Dal punto di vista della confezione complessiva e del sistema di sostegni il caso più vicino a quello di Lipari sembra essere il chiostro del monastero benedettino di San Venerio sull'isola del Tino, probabilmente dell'inizio del XII secolo: A. FRONDONI, Architettura ecclesiastica al Tino: $i$ dati archeologici, in S. Venerio del Tino: vita religiosa e civile tra isole e terraferma in età medievale, Atti del convegno (Lerici-La Spezia-Portovenere, 18-20 settembre 1982), La Spezia-Sarzana, 1986, p. 143-177; EAD., Archeologia all'Isola del Tino: il monastero di San Venerio, Genova, 1995; F. CERVINI, op. cit. (n. 69), p. 225.

77 R. LEGLER, op. cit. (n. 42), p. 231-234. 